\title{
Permafrost in der oberen subnivalen Stufe der Alpen
}

\author{
Dietrich Barsch
}

Bisher ist unseres Wissens die Frage nach dem Vorkommen von rezentem Permafrost in den Alpen nie eindeutig beantwortet worden. Bei unseren Untersuchungen im Jura (D. Barsch, 1967) sind wir verschiedentlich auf Erscheinungen gestoßen, die nur unter der Annahme von würmzeitlichem Permafrost befriedigend erklärt werden können. Für den Jura beweisen besonders die "periglazialen Seen» (D. Barsch, 1968), die in den würmzeitlich durch Permafrost blockierten Karstwannen bestanden haben, die Existenz von lückenlosem würmzeitlichem Permafrost dicht unter der damaligen Schneegrenze. $\mathrm{Er}$ dürfte in der damaligen oberen subnivalen Höhenstufe des Juras der rezenten «continuous permafrost zone» der amerikanischen Geologen in Alaska entsprochen haben. Aus naheliegenden Gründen müßte deshalb auch in der heutigen subnivalen Höhenstufe der Alpen rezenter Permafrost zu erwarten sein. Bisher sind uns dazu zwei Beispiele bekannt geworden, die wir hier in einer vorläufigen Mitteilung veröffentlichen wollen. Wir hoffen, daß damit auch andere angeregt werden, nach weiteren Hinweisen zu suchen. Es scheint angezeigt, den Problemen, die mit dem rezenten Auftreten von Permafrost in den Alpen zusammenhängen, im Rahmen einer größeren Arbeitsgemeinschaft nachzugehen.

Beim Bau der Berg- und der Mittelstation der Corvatschbahn (Oberengadin) sowie beim Aushub für einige der Stützenfundamente der Bahn wurden Spaltenfüllungen aus Eis entdeckt ${ }^{1}$. Nach freundlicher brieflicher Mitteilung der Bahnverwaltung hat es sich vor allem bei der Bergstation und bei einigen Stützenfundamenten um ein Fels-Eis-Gemisch gehandelt. Bei der Bergstation fand man Eis bis zur soliden Felssohle in rund $15 \mathrm{~m}$ Tiefe. Beim Aushub für die Mittelstation, der allerdings bereits im Frühjahr (März bis Mai) durchgeführt wurde, konnten bis in rund $4 \mathrm{~m}$ Tiefe Eislinsen und gefrorener Schutt festgestellt werden. Obwohl keine genaueren Aufnahmen vorliegen, ist kein Zweifel mehr möglich: Im Gebiet der Bergstation der Corvatschbahn tritt Permafŗost auf, im Gebiet der Mittelstation ist das Vorkommen von Permafrost sehr wahrscheinlich.

Die Bergstation der Corvatschbahn liegt nördlich des gleichnamigen Gipfels $(3451 \mathrm{~m})$ in $3304 \mathrm{~m}$ Höhe, die Mittelstation in $2699 \mathrm{~m}$ Höhe. Die rezente Waldgrenze dürfte zwischen 2200 und
2400 m (F. Holtmeier, 1967), die rezente Schneegrenze bei etwa $3000 \mathrm{~m}$ anzusetzen sein. Allerdings hängt gerade die Lage der lokalen Schneegrenze im Oberengadin stark von den topographischen Verhältnissen ab. So apert der Grat, auf dem die Bergstation erbaut ist, trotz seiner großen Höhenlage jedes Jahr aus. Wir befinden uns also an dieser Stelle im Grenzbereich zwischen der nivalen und der subnivalen Zone. Hier ist - entsprechend den besonderen Verhältnissen - das Auftreten von Permafrost durchaus verständlich. Frost tritt in dieser Höhe das ganze Jahr über auf und selbst für die Sommermonate ist mit einem negativen Temperaturmittel zu rechnen. Außerdem ist, nach Beobachtungen in Alaska und in Sibirien, der Permafrost überall dort sehr mächtig, wo eine fehlende Schneedecke das Eindringen des Frostes in den Boden begünstigt. Noch interessanter ist das wahrscheinliche Auftreten von Permafrost im Bereich der Mittelstation der Corvatschbahn, da dieses Gebiet deutlich unterhalb der Schneegrenze im oberen Teil der subnivalen Höhenstufe der Alpen liegt. Daß Permafrost auch in dieser Höhe generell möglich ist, zeigt der Blockgletscher, der sich nur wenige hundert Meter südwestlich der Station zwischen 2730 und $2880 \mathrm{~m}$ Höhe entwickelt hat. Nach unseren Untersuchungen in Macun (s. u.) bestehen die Blockgletscher fast vollkommen aus gefrorenem Schutt, und nur die oberste Blockschicht besteht aus ungefrorenem Material. Wir folgern daraus, daß das Auftreten von Permafrost im Bereich der Mittelstation der Corvatschbahn nicht unwahrscheinlich ist.

Ein weiteres Beispiel für rezenten Permafrost stammt aus dem Unterengadin. Im Rahmen eines Forschungsprogramms des Geographischen Instituts der Universität Basel haben wir im Großkar von Macun (südlich Lavin, Unterengadin) seit 1965 die Blockgletscher untersucht. Mit einer Arbeitsgruppe von Studenten wurden geomorphologische Detailkartierungen und mit Hilfe des Vermessungsfachmannes M. Maurer das Bewegungsbild der Blockgletscher Macun 1 und Macun 2 für die Zeiträume 1965-1967, 1967-1968 bestimmt. Ein erster Bericht über die Ergebnisse ist seit einiger Zeit im Druck (Zeitschrift für Geomorphologie). Unsere Studien zum Bewegungsvorgang der Blockgletscher mußten unvollständig bleiben, wenn nicht weitere Angaben über den inneren Aufbau der Blockgletscher gesammelt werden konnten. Mit Hilfe der 
großzügigen Unterstützung durch den Schweizerischen Nationalfonds zur Förderung der wissenschaftlichen Forschung, dem auch an dieser Stelle herzlich gedankt sei, war es möglich, die notwendigen Daten zu gewinnen: Durch die Firma Geotest AG, Bern (Dr. A. Schneider), sowie unter Einsatz unserer Arbeitsgruppe aus Studierenden haben wir den genau vermessenen und in seinen äußeren Bewegungsstrukturen gut bekannten Blockgletscher Macun 1 mittels Sprengseismik erkundet. Dabei ergab schon die vorläufige Auswertung, daß - abgesehen von der obersten Blockschicht - der gesamte Blockgletscher als starre Masse reagiert. Die Druckwellen hatten im Blockgletscher die Geschwindigkeit wie in Eis. Aufgrabungen an den seitlichen Bewegungsstirnen im oberen Teil des Blockgletschers haben zudem gezeigt, daß dort stellenweise schon in rund $1 \mathrm{~m}$ Tiefe festgefrorenes Material ansteht. Feinmaterial und gröbere Fragmente bilden eine beinharte, von dünnen Eislinsen durchzogene Masse, die als Eiszement bezeichnet wird. Im allgemeinen tritt das Eis nur in dünnen Häutchen an den gröberen Fragmenten auf; größere Eislinien sind sehr selten.

An der Oberfläche des Blockgletschers konnte zudem verschiedentlich fließendes Schneeschmelzwasser (August!) beobachtet werden. Wir haben an diesen Stellen die groben Blöcke weggeräumt und dabei festgestellt, da $\beta$ das Wasser auf Grundeis fließt und deshalb nicht versickern kann. Alle Gesteinsfragmente sind an der Sohle der Gerinne festgefroren. Damit ist unseres Wissens zum ersten Mal exakt bewiesen worden, daß das Innere der aktiven Blockgletscher aus einem festgefrorenen Schuttkörper besteht, der durch sein Eigengewicht und das Gewicht der Blockschicht im unteren Teil plastisch wird. Dieser gefrorene Schuttkörper ist für den Fließvorgang verantwortlich.

$\begin{array}{ll}\text { Höhe } & \text { Station } \\ 3579 & \text { Jungfraujoch } \\ 3488 & \text { Plateau Rosa } \\ & \text { (Testa Grigia) } \\ 2667 & \text { Weißfluhjoch } \\ 2479 & \text { Grand St-Bernard } \\ 2237 & \text { Julier Hospiz } \\ 2096 & \text { St. Gotthard } \\ 1968 & \text { Buffalora } \\ 1853 & \text { St. Moritz II }\end{array}$

Periode
ab 1938
ab 1953
ab 1947
ab 1818
$1864-1910$
ab 1903
ab 1917
1932-1953

Die Stationen, die ungefähr in der fraglichen Höhenstufe um 2600 bis $3000 \mathrm{~m}$ liegen, weisen nur vier Monate mit deutlich positiver Mitteltemperatur auf. Das Maximum der monatlichen Mitteltemperaturen beträgt auf demWeißfluhjoch $4,7^{\circ} \mathrm{C}$ (August), auf dem Großen St. Bernhard $6,8^{\circ} \mathrm{C}$ (August).
In Macun umfaßt der Blockgletscher Macun 1 den Höhenbereich zwischen 2600 und $2720 \mathrm{~m}$. Die Waldgrenze dürfte hier bei $2200-2300 \mathrm{~m}$, die Schneegrenze bei etwa 2950-3000 m anzunehmen sein. Die Blockgletscher, d. h. die Zonen gefrorenen Schuttes, liegen deutlich unter der Schneegrenze im oberen Teil der subnivalen Höhenstufe. Wir deuten die Blockgletscher deshalb als eine Form des periglazialen und nicht des glazialen Bereiches; der in ihnen vorhandene und für ihre Bewegung wesentliche, gefrorene Schutt ist so als eine besondere Erscheinungsform des Permafrostes anzusehen.

Aus unseren beiden Beispielen geht hervor, daß im oberen Teil der subnivalen Höhenstufe in den Alpen mit der Existenz von Permafrost zu rechnen ist. Wir wissen bisher nicht, welche Verbreitung dieses Phänomen in den Alpen besitzt, doch dürfte es häufiger auftreten, als bisher bekannt ist. Eine $\mathrm{Zu}-$ ordnung zu einzelnen Zonen nach der Intensität der Bildung (lückenlos, lückenhaft, sporadisch) ist bisher noch nicht möglich. Ebensowenig ist bisher entschieden, ob es in den Alpen auch den sogenannten "dry permafrost» gibt. Man versteht darunter wasserlose beziehungsweise eisfreie Gesteinspartien, die während des ganzen Jahres Temperaturen unter dem Gefrierpunkt aufweisen. Vielleicht können systematische Beobachtungen aller neuen Bauvorhaben in den Alpen beziehungsweise Auswertungen der häufig lokal archivierten Beobachtungen, die anläßlich früherer Bauten gesammelt worden sind, weiterhelfen.

Von seiten der klimatischen Verhältnisse, über die wir auf Grund des mangelnden Stationennetzes nur wenige Angaben für die Schweizer Alpen machen können, scheint ebenfalls die Existenz von Permafrost im Bereich der oberen subnivalen Höhenstufe im Engadin möglich. Nach M. Schüepp (1960) habe ich die folgenden Angaben zusammengestellt, die alle auf die Periode 1901 - 1940 bezogen sind:

$\begin{array}{rrrrr}\text { Winter } & \text { Frühling } & \text { Sommer } & \text { Herbst } & \text { Jahr } \\ -14.1 & -10.5 & -2.4 & -7.1 & -8.5 \\ -12.2 & -8.6 & 0.1 & -5.0 & -6.4 \\ & & & & \\ -8.9 & -4.9 & 3.8 & -1.3 & -2.8 \\ -8.2 & -3.6 & 5.8 & -0.6 & -1.7 \\ -8.1 & -1.9 & 7.3 & 0.1 & -0.7 \\ -6.9 & -1.8 & 7.0 & 0.7 & -0.2 \\ -9.7 & -0.8 & 9.0 & 0.7 & -0.2 \\ -6.1 & 1.5 & 10.3 & 2.8 & 2.1\end{array}$

Fröste können zu allen Jahreszeiten auftreten. Der Einfluß der negativen Temperaturen überwiegt. Daraus folgt, $\mathrm{da} \beta$ die thermischen Voraussetzungen für die Bildung von Permafrost im Höhenbereich der oberen subnivalen Stufe im Gebiet des Engadins durchaus gegeben sind. 


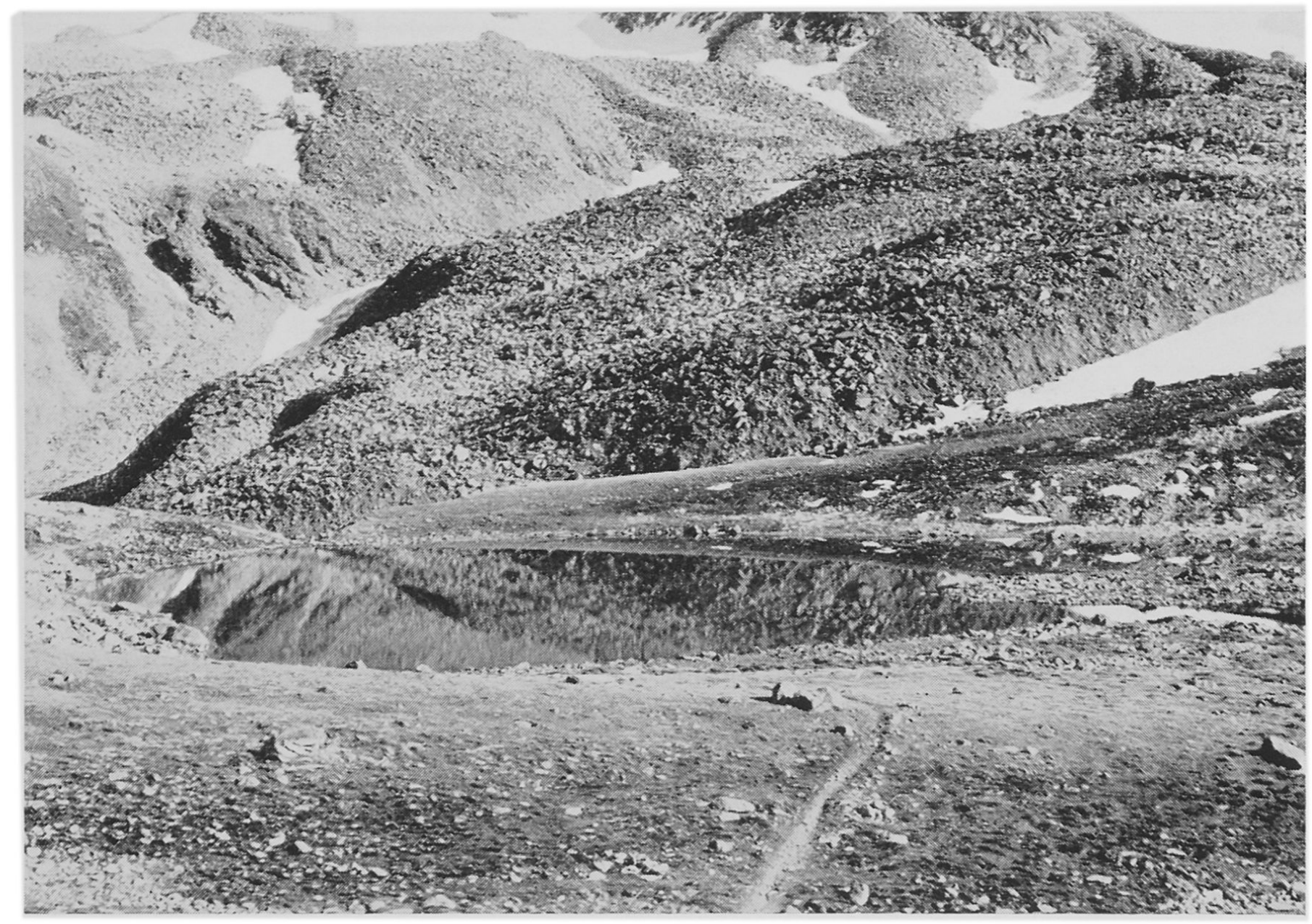

Blockgletscher in Macun (Unterengadin). Photo J. Rohner

\section{Literatur}

Barsch, D. (1967): Studien zur Geomorphogenese des zentralen Berner Juras. Manuskript 185 p. Erscheint als Heft 9 der Basler Beiträge zur Geographie.

Barsch, D. (1968): Periglaziale Seen in den Karstwannen des Schweizer Juras. Regio Basiliensis 9, p. $115-134$.

Barsch, D. (im Druck): Studien und Messungen an Blockgletschern in Macun (Unterengadin). Zeitschrift für Geomorphologie. (Manuskript abgeschlossen im April 1968.)

Holtmeier, F. (1967): Die Verbreitung der Holzarten im Oberengadin unter dem Einfluß des Menschen und des Lokalklimas. Erdkunde 21, p. 249258.

Schüepp, M. (1960): Klimatologie der Schweiz. Teil
C, 1: Lufttemperatur. Beiheft zu den Annalen der Schweizerischen Zentralanstalt (Jahrgang 1959). Zürich.

\section{Anmerkung}

${ }^{1}$ Einen ersten Hinweis auf diese Verhältnisse erhielt ich von Herrn PD Dr. V. Trommsdorff (Mineralogisch-Petrographisches Institut der Universität Basel). Die Erscheinungen wurden mir brieflich durch die Bahnverwaltung bestätigt. Der Direktor der Bahn, Herr Rohrer, besorgte mir außerdem die Anschrift des damaligen Bauführers, Herrn Camadeni, der mir vor allem die Verhältnisse an der Mittelstation beschrieben hat. Ich möchte auch an dieser Stelle allen Genannten für ihre Auskünfte herzlich danken. 